\title{
Low-grade periprosthetic knee infection: diagnosis and management
}

\author{
Michele Vasso $\cdot$ Alfredo Schiavone Panni
}

Received: 27 December 2013/Accepted: 29 March 2014/Published online: 14 May 2014

(C) The Author(s) 2014. This article is published with open access at Springerlink.com

\begin{abstract}
Diagnosis and management of low-grade periprosthetic knee infection are still controversial and debatable. The diagnosis of low-grade infection after total knee arthroplasty is often complex, as clinical symptomatology and diagnostic studies are highly conflicting and knees often exhibit well-fixed components. Although the criterion standard for staged reimplantation is interim placement of an antibiotic-loaded spacer, less-invasive surgical procedures have been advocated for managing infections caused by lowvirulence bacteria. Debridement with polyethylene exchange and single-stage reimplantation could offer advantages, such as fewer surgeries, reduced potential for intraoperative complications, and lower direct social costs. The aim of this narrative review was to analyze the literature to evaluate the effectiveness of different surgical procedures in managing low-grade periprosthetic knee infections. Additionally, the most reliable investigations for diagnosing total knee infection caused by low-virulence bacteria were reviewed.

Level of evidence Level V.
\end{abstract}

Keywords Total knee arthroplasty - Low-grade infection · Diagnosis · Debridement · Reimplantation

\section{Introduction}

Infection continues to be a rare but devastating complication of total knee arthroplasty (TKA), occurring in 1-3\% of cases [1-5]. Despite the low incidence, infection is associated with patient morbidity, increased healthcare costs, and recurrence

M. Vasso $(\varangle) \cdot$ A. Schiavone Panni

Department of Medicine and Science for Health, University of

Molise, Via Francesco De Sanctis, Campobasso, Italy

e-mail: vassomichele@gmail.com and is also challenging to control [6]. TKA infections are often attributable to staphylococci and streptococci, whereas aerobic gram-negative bacteria cause $10-20 \%$ of all infections and anaerobic bacteria are responsible for another $10 \%$ [7]. Methicillin-sensitive staphylococci, streptococci, and anaerobic cocci are commonly considered as low-virulence bacteria causing low-grade periprosthetic infections; methicillin-resistant staphylococci, enterococci, and gram-negative organisms are certainly considered bacteria of high virulence due to their intrinsic resistance to antimicrobial agents and antibiotics [8]. The diagnosis of a TKA infection, itself very challenging, becomes highly complex in the presence of low-virulence organisms, as clinical features and diagnostic tests may be conflicting. Common clinical signs of infection are often absent, and a gold standard for preoperative diagnosis does not exist.

Whereas two-stage reimplantation is considered worldwide to be the most successful procedure in treating TKA infections, regardless of the etiology of the infecting organism(s) (and timing of infection) [2, 4, 6, 9-12], treatment of a low-grade prosthetic knee infection remains controversial and debatable. Less-invasive and more viable surgical procedures have been advocated for managing lowgrade TKA infections (especially if early): debridement with insert exchange and single-stage reimplantation has the advantage of less surgery, ability to maintain motion and soft-tissue health, and lower costs. Furthermore, these procedures mean that the patient is never without prosthetic components, clearly improving comfort [1, 12-14]. Regardless of the virulence of the infecting organisms, debridement and component retention results in inconsistent infection control rates of 16-80\% [15], whereas singlestage revision, in which new, sterile components are implanted and secured with antibiotic-loaded bone cement, presents a large variability in successful infection control of 
$73-100 \%[6,15]$. However, few data exist about the success rates of these different treatments strategies in TKA infections caused by low-virulence bacteria. Therefore, the purpose of this review was to analyze the literature to determine the most effective surgical treatment for managing low-grade periprosthetic knee infection. We also reviewed the most reliable investigations for diagnosing TKA infection caused by low-virulence bacteria.

\section{Diagnosing low-grade periprosthetic knee infection}

Diagnosing low-grade infection after TKA is often highly complex, as clinical symptomatology and diagnostic studies may be conflicting. Moreover, patients often present with well-fixed components, even in in acute infections. We believe the diagnostic process should be developed using the following steps.

History and clinical features

Early postoperative low-grade TKA infections generally present only with moderate knee pain and stiffness and/or difficult and delayed rehabilitation; persistent fever, severe pain, local warmth, erythema, and swelling are often absent. Hematogenous low-grade infection is quite rare and characterized by a sudden and unexpected deterioration in a previously well-functioning joint. There may also be a history of acute illness followed by sudden deterioration in knee function, with fever and chills $[14,16]$. Chronic lowgrade TKA infections do not show evident onset. Classic presentation with pain, fever, and local signs such as sinus tract, redness, and swelling is uncommon. More frequently, patients claim only moderate pain and stiffness, which exist since knee replacement $[14,16]$.

\section{Radiographs}

Standard X-rays are not very useful in the diagnosis of lowgrade infection after TKA, particularly in acute infections in which X-rays are always normal. Radiographs showing periosteal new bone formation, scattered foci of osteolysis, and subchondral bone resorption are highly suggestive of infection but typically may be late findings. Periprosthetic radiolucency may be unrelated to a septic process, and serial radiographs help rule out other conditions, such as wear, osteolysis, or fracture [16].

\section{Laboratory findings}

Peripheral white blood cell (WBC) count is frequently normal in low-grade TKA infections and affords little diagnostic help [17]. Erythrocyte sedimentation rate (ESR) and C-reactive protein (CRP) remain the most useful laboratory investigation, even though their sensitivity may be diminished [19]. An ESR $>30 \mathrm{~mm} / \mathrm{h}$ or CRP $>10 \mathrm{mg} / \mathrm{L}$ should be considered abnormal $[7,18]$. Due to this lower sensitivity, serum interleukin-6 (IL-6) could represent a more reliable marker of periprosthetic low-grade infection $[17,19]$. With a threshold of $<10 \mathrm{pg} / \mathrm{ml}$, serum IL-6 test shows sensitivity, specificity, positive predictive value (PPV), negative predictive value (NPV), and accuracy of $1.0,0.95,0.89,1.0$, and $97 \%$, respectively, thus being a more accurate marker than ESR and CRP [17, 19]. Due to its excellent sensitivity, IL-6 could be an optimal tool for diagnosing low-grade TKA infection [16, 19].

\section{Radionucleotide scanning}

Technetium-99 bisphosphonate scan in conjunction with indium-111-labeled leukocyte scan can contribute to the diagnosis of low-grade TKA infection. Technetium scan is quite reliable in detecting bone-remodelling changes around prosthetic components; however, when positive, it cannot distinguish between aseptic loosening and infection [22]. It has a low NPV in low-grade infections: a normal scan suggests that loosening is not the likely cause of pain, but it does not rule out the possibility of infection [16]. Leukocyte scan is more sensitive but has low specificity. Combining a leukocyte with a technetium scan improves the accuracy for detecting low-grade infection [16]. In particular, if uptake on leukocyte scan is more intense than on technetium scan, it is probable that the TKA is infected. Isotope scanning may present false-positive results: within the first postoperative year, increased scan activity may be present around $85-90 \%$ of tibial and $60-65 \%$ of femoral components in asymptomatic knees [23].

\section{Aspiration}

In the setting of a low-grade infection, knee aspiration could present poor sensitivity due to the low bacterial load. Antibiotic therapy must be suspended at least 2 weeks before aspiration to avoid further false-negative results [18]. The aspirate should be sent for aerobic, anaerobic, and fungal cultures. If the first aspirate is negative, then at least two additional aspirations should be performed [23]. Specimens obtained from the joint must be separated into two or three samples: if all samples are positive for the same organism, the aspiration is considered positive; if only one sample is positive or presents an unexpected positive result, aspiration must be repeated given the high suspicion of contamination [24]. Synovial-fluid WBC count and differential are two helpful parameters: cutoff values for optimal accuracy iare $>1,100$ cells $/ \mathrm{mm}^{3}$ for fluid leukocyte count and $>64 \%$ for neutrophil differential [25]. 
Molecular tests

The detection of bacterial DNA or RNA in synovial fluid using polymerase chain reaction (PCR) studies could be helpful in low-grade TKA infections in which bacterial load is low. PCR amplifies strains of bacterial (deoxy) RNA and can detect nonviable bacteria that do not grow on culture as well as bacteria lysed by ultrasonication, providing results within $12-13 \mathrm{~h}$. Results of PCR are unaffected by the administration of antibiotics [16]. However, molecular techniques cannot distinguish between live or dead organisms, generating false-positive results (low specificity) and therefore being of little clinical utility [26].

Intraoperative histopathology

Gram staining of intraoperative specimens obtained from the joint capsule or periprosthetic membrane has been reported as being of little help in diagnosing low-grade TKA infection [27]. Intraoperative frozen section for identifying neutrophils in periprosthetic tissue is used to help intraoperative decision making. The exact histologic criteria used for diagnosing infection are not uniform. However, five to ten polymorphonuclear leukocytes per high power field $(\times 400)(\mathrm{PMNs} / \mathrm{HPF})$ in at least five fields is considered consistent with infection $[7,16]$. This method is highly dependent on the tissue selected and interpretation by the pathologist. The poor sensitivity of this technique in low-grade infection is probably due to the low inflammatory response caused by coagulase-negative staphylococci, the organisms most commonly infecting TKA [7].

\section{Sonication}

At the time of prosthesis removal, sonication could improve sensitivity of microbiological investigations that, as mentioned above, is significantly diminished in lowgrade infections [28]. Sonication uses ultrasound (US) energy to mechanically disrupt biofilm on removed implants following revision surgery. This increases the number of bacteria isolated on culture, or molecular techniques enabling detection of bacteria that would have been missed by conventional tissue culture [28]. Improvement in sensitivity is particularly notable in TKA infected by lowvirulence organisms and in patients on antibiotics within 2 weeks prior to surgery [21].

\section{Surgical management of low-grade periprosthetic knee infection}

The goal of treatment is infection eradication and maintenance of a pain-free and functional joint. Different treatment options have been advocated: suppressive antibiotic therapy, irrigation and debridement, single-stage reimplantation, two-stage reimplantation, salvage procedures, and above-knee amputation. Whereas two-stage exchange is considered the best approach regardless of the infecting organism virulence and timing of infection $[2,4$, 6, 9-12], optimal management of low-grade TKA infection remains controversial, as open debridement with insert exchange and single-stage reimplantation have been proposed as valid surgical alternatives with the least impact. Suppressive antibiotic therapy has been reserved for patients medically unable to undergo surgery, with limited success rates in low-grade infection [11, 16]. Salvage procedures, such as resection arthroplasty or arthrodesis, and amputation have never been necessary in TKA infected by sensitive bacteria [14]. Therefore, open debridement and single- and two-stage reimplantation are the only procedures reviewed in this paper.

Irrigation and debridement

In the setting of a low-grade TKA infection, debridement with component retention, and local and systemic antibiotic application could be indicated in healthy patients affected by acute (early postoperative and hematogenous) gram-positive infection with a stable and well-functioning prosthesis and good soft tissue envelope with no fistula [8, 28]. When attempting component retention, thorough debridement and rapid antibiotic treatment prior to the accumulation of biofilm are paramount for a successful outcome [16, 29, 30]. Contraindications are chronic infection, implant loosening, poor soft tissue envelope, and patients with other arthroplasties or a defective heart valve $[16,28]$. Polyethylene exchange is always preferred, as it allows complete synovectomy and better debridement of the posterior synovium, and eliminates biofilm formation on the polyethylene [29, 31]. One reason for the failure of arthroscopic debridement is likely due to the inability to eliminate biofilm at the polyethylene-prosthesis interface and debride the posterior aspect of the knee [28, 29]. Intraoperative cultures are performed on synovial fluid and membrane, infected tissues, and polyethylene-implant interface. Organism-specific intravenous antibiotic application is initiated for $4-6$ weeks, followed by protracted oral antibiotic administration.

Single-stage reimplantation

Low-grade infection after TKA has been considered to be potentially susceptible to a single-stage revision due to the low virulence of the infecting bacteria [5, 32, 33]. Factors associated with successful single-stage reimplantation include pathogen identification before revision, infections 
caused by gram-positive bacteria, absence of sinus tract, and use of antibiotic-loaded bone cement for new component fixation [34]. Single-stage reimplantation involves explantation of all components and cement, thorough debridement, copious irrigation, and reimplantation of new and appropriate prosthetic components with antibioticimpregnated cement, followed by $6-12$ weeks of systemic antibiotic therapy. Then, oral antibiotic therapy should be considered for 3-6 additional months based on recommendations [32]. In low-grade infection, single-stage revision may be advantageous, decreasing recovery time and costs and avoiding some of the problems of two-stage procedures, such as stiffness and arthrofibrosis resulting from a period with a spacer in situ. Furthermore, debridement and a single-stage strategy allows the patient to retain their prosthetic components [13].

\section{Two-stage reimplantation}

Two-stage reimplantation is actually considered state of the art for treating both acute and chronic TKA infections, with reported success rates of $88-96 \%$ regardless the etiology of the infecting organism [2, 4, 6, 9-12]. During the first stage, removal of all components and cement, complete synovectomy, and debridement of all necrotic and infected tissue are performed. Multiple specimens are obtained from deep synovial biopsies and sent for aerobic, anaerobic, and fungal cultures. Resected bone ends and joint space are thoroughly irrigated with pulsatile lavage. Successively, an antibiotic-impregnated cement spacer is positioned into the joint $[6,12,35,36]$. In low-grade infection, $2 \mathrm{~g}$ of vancomycin for a $40-\mathrm{g}$ pack of bone cement could be sufficient $[18,37]$, as the majority of preformed spacers contain gentamicin. The choice of antibiotic should depend upon the antibiogram: in fact, even though virulence is low, antibiotic susceptibility might be low also [38]. Between stages, targeted intravenously and orally administered antibiotics are generally used for 6-12 weeks on the basis of recommendations of an infectious disease consultant [1, 10].

Decision for second-stage reimplantation is made after a minimum 2-week antibiotic-free interval, when clinical signs of infection have subsided and ESR and CRP levels have steadily trended toward to normal $[18,37]$. In lowgrade infection, laboratory markers may also normalize in patients with persistent infection, so that knee aspiration before reimplantation should be performed in patients with suspected persistent infection [18, 20, 39]. The second stage includes explantation of the cement spacer, removal of all cement fragments, thorough debridement of the joint and intramedullary canals, copious irrigation, and placement of the appropriate new prosthetic components fixed with vancomycin-impregnated bone cement [18, 40, 41].
After reimplantation, patients receive antibiotics intravenously until intraoperative cultures return to normal.

\section{Results}

In the setting of low-grade TKA infections, irrigation and debridement has shown unsatisfactory success rates, ranging on average from $16 \%$ to $70 \%[8,14]$. In acute (early postoperative or hematogenous) infections, primary open debridement is reported as successful in $56 \%$ of patients infected by low-virulence organisms, such as Staphylococcus epidermidis or Streptococcal species, but only in $8 \%$ of patients infected by S. aureus [42]. Assessing 247 knees, Buller et al. [31] reported an overall $45 \%$ failure rate of debridement; higher failure rates were found in infections by resistant organisms and in patients with symptoms $\geq 21$ days. A $34 \%$ failure rate was also reported in low-grade infections. Barberan et al. [43] reported an overall debridement failure rate of $35 \%$ in low-grade infections, which ranged from $17 \%$ in patients with symptom duration $<1$ month to $69 \%$ in patients with symptoms duration $>6$ weeks. Better results were reported when considering only early postoperative (and not hematogenous) infections: Kim et al. [44] reported that 27 of 32 knees $(84 \%)$ were treated successfully with perioperative debridement.

Certainly, debridement is reportedly unsuccessful for treating chronic low-grade infections, with a final failure rate of $100 \%[8,29,45]$. The most compelling evidence to discourage the use of debridement in low-grade infections is the $34 \%$ failure rate of two-stage reimplantation after failed irrigation and debridement [8]. Fehring et al. [8] suggested limiting its use to early postoperative infections, in which the date of inoculation is well defined and perioperative debridement should improve infection control because intervention may occur before the establishment of drug-resistant biofilm on the implant or before osteomyelitis becomes entrenched in periprosthetic bone.

Reports related to single-stage revision are generally sparse and of poor quality [5]. Single-stage reimplantation has been successful in highly selected cases or small series, with an average success rate of $81 \%$ [5, 12, 15]. In lowgrade infections, reinfection rates are reported ranging from $5 \%$ to 11 , although in studies with small case series and short follow-ups [5, 11, 13, 34]. Silva et al. [34] reported a success rate of $89 \%$ and found that factors associated with success are absence of sinus formation, gram-positive infection, use of antibiotic-impregnated bone cement in reimplantation, and 12 weeks of antibiotic therapy. Baker et al. [13] reported no significant differences between single- and two-stage revision in terms of postoperative knee score, general health perception, or 
satisfaction in patients with low-grade TKA infection. They concluded that single-stage treatment may be functionally superior in cases in which infection is successfully eradicated but be may be prone to higher rates of reinfection, which are associated with poorer outcomes. Singer et al. [5] reported that single-stage revision achieved a control rate of $95 \%$ in low-grade TKA infections in which patients with methicillin-resistant $S$. aureus (MRSA), methicillin-resistant $S$. epidermidis (MRSE), or unknown microorganisms were excluded from the study. Higher rates of recurrent infection appeared to be associated with long-term chronic infections of hinged prostheses. Given the very limited number of reliable studies assessing the efficacy of single-stage reimplantation in eradicating lowgrade infection following TKA, further investigations are warranted [12].

The success rates of two-stage reimplantation in eradicating infection and restoring function are almost constantly reported to be $>90 \%$, regardless of the etiology of infecting organisms and the timing of infection $[2,4,6,9$ 12], so that this procedure is now considered the most reliable treatment option for infected TKA. Additionally, two-stage reimplantation is reported to be even more successful when used in low-grade TKA infections $[2,4,9,39$, 46-48]. Volin et al. [48] reported a $94.6 \%$ success rate in methicillin-sensitive $S$. aureus infections. Salgado et al. [47] reported a $17 \%$ failure rate for treated MRSA infections, whereas no failures were noted among infections caused by methicillin-sensitive $S$. aureus. Cordero-Ampuero et al. [9] reported that infection was eradicated in 22 of $25(88 \%)$ patients infected by methicillin-resistant staphylococci and in 14 of 14 (100\%) infected by methicillinsensitive staphylococci.

\section{Conclusions}

The diagnosis of a TKA infection, which in itself is highly challenging, may be extremely complex in the presence of low-virulence organisms. Clinical features are often conflicting, whereas classic presentation of pain, fever, and local signs such as sinus tract, redness, and swelling is uncommon. ESR and CRP levels remain the first-line investigation, even though the sensitivity of their results is often diminished in low-grade infections. In these cases, IL-6 levels should support ESR and CRP evaluation to significantly increase the sensitivity of the laboratory findings. Technetium and leukocyte scanning should both be used, and prosthesis sonication may help detect bacteria missed by conventional tissue culture. In patients with an acute postoperative low-grade infection, the strategy of irrigation and debridement with insert exchange persists given the emotional investment in dealing with this complication by both patient and surgeon. Therefore, an attempt to "save the implant" through open debridement appears well intentioned despite a high reported failure rate. In the event of failed debridement or of chronic infections, resection of all components is necessitated. A single-stage exchange has the potential to decrease the number of surgeries and, subsequently, costs. However, infection eradication rates of direct exchange show this method is less safe and predictable than the two-stage revision. This suggests that two-stage reimplantation, with placement of an intrastage antibiotic-loaded spacer, should represent the gold standard for managing low-grade infections.

Conflict of interest The authors declare that they have no conflict of interest.

Open Access This article is distributed under the terms of the Creative Commons Attribution License which permits any use, distribution, and reproduction in any medium, provided the original author(s) and the source are credited.

\section{References}

1. Johnson AJ, Sayeed SA, Naziri Q, Khanuja HS, Mont MA (2012) Minimizing dynamic knee spacer complications in infected revision arthroplasty. Clin Orthop Relat Res 470:220-227

2. Kurd MF, Ghanem E, Steinbrecher J, Parvizi J (2010) Two-stage exchange knee arthroplasty: does resistance of the infecting organism influence the outcome? Clin Orthop Relat Res 468: 2060-2066

3. Laudermilch DJ, Fedorka CJ, Heyl A, Rao N, McGough RL (2010) Outcomes of revision total knee arthroplasty after methicillin-resistant Staphylococcus aureus infection. Clin Orthop Relat Res 468:2067-2073

4. Siddiqui MM, Lo NN, Ab Rahman S, Chin PL, Chia SL, Yeo SJ (2013) Two-year outcome of early deep MRSA infections after primary total knee arthroplasty: a joint registry review. J Arthroplast 28:44-48

5. Singer J, Merz A, Frommelt L, Fink B (2012) High rate of infection control with one-stage revision of septic knee prostheses excluding MRSA and MRSE. Clin Orthop Relat Res 470: 1461-1471

6. Jaekel DJ, Day JS, Klein GR, Levine H, Parvizi J, Kurtz SM (2012) Do dynamic cement-on-cement knee spacers provide better function and activity during two-stage exchange? Clin Orthop Relat Res 470:2599-2604

7. Bori G, Soriano A, Garcia S, Mallofrè C, Riba J, Mensa J (2007) Usefulness of histological analysis for predicting the presence of microorganisms at the time of reimplantation after hip resection arthroplasty for the treatment of infection. J Bone Joint Surg Am A 89:1232-1237

8. Fehring TK, Odum SM, Berend KR, Jiranek WA, Parvizi J, Bozic KJ, Della Valle CJ, Gioe TJ (2013) Failure of irrigation and débridement for early postoperative periprosthetic infection. Clin Orthop Relat Res 471:250-257

9. Cordero-Ampuero J, Esteban J, Garcia-Rey E (2010) Results after late polymicrobial, gram-negative, and methicillin-resistant infections in knee arthroplasty. Clin Orthop Relat Res 468:1229-1236 
10. Kalore NV, Maheshwari A, Sharma A, Cheng E, Gioe TJ (2012) Is there a preferred articulating spacer technique for infected knee arthroplasty? A preliminary study. Clin Orthop Relat Res 470:228-235

11. Masters JP, Smith NA, Foguet P, Reed M, Parsons H, Sprowson AP (2013) A systematic review of the evidence for single stage and two stage revision of infected knee replacement. BMC Musculoskelet Disord 14:222

12. Romanò CL, Gala L, Logoluso N, Romanò D, Drago L (2012) Two-stage revision of septic knee prosthesis with articulating knee spacers yields better infection eradication rate than onestage or two-stage revision with static spacers. Knee Surg Sports Traumatol Arthrosc 20:2445-2453

13. Baker P, Petheram TG, Kurtz S, Konttinen YT, Gregg P, Deehan D (2013) Patient reported outcome measures after revision of the infected TKR: comparison of single versus two-stage revision. Knee Surg Sports Traumatol Arthrosc 21:2713-2720

14. Chun KC, Kim KM, Chun CH (2013) Infection following total knee arthroplasty. Knee Surg Relat Res 25:93-99

15. Parvizi J, Zmistowski B, Adeli B (2010) Periprosthetic joint infection: treatment options. Orthopedics 33:659

16. Kalore NV, Gioe TJ, Singh JA (2011) Diagnosis and management of infected total knee arthroplasty. Open Orthop J 5:86-91

17. Berbari E, Mabry T, Tsaras G, Spangehl M, Erwin PJ, Murad MH, Steckelberg J, Osmon D (2010) Inflammatory blood laboratory levels as markers of prosthetic joint infection: a systematic review and meta-analysis. J Bone Joint Surg Am 92:2102-2109

18. Kusuma SK, Ward J, Jacofsky M, Sporer SM, Della Valle CJ (2011) What is the role of serological testing between stages of two-stage reconstruction of the infected prosthetic knee? Clin Orthop Relat Res 469:1002-1008

19. Garvin KL, Konigsberg BS (2012) Infection following total knee arthroplasty: prevention and management. Instr Course Lect 61:411-419

20. Ghanem E, Azzam K, Seeley M, Joshi A, Parvizi J (2009) Staged revision for knee arthroplasty infection: what is the role of serologic tests before reimplantation? Clin Orthop Relat Res 467:1699-1705

21. Savarino L, Tigani D, Baldini N, Bochicchio V, Giunti A (2009) Pre-operative diagnosis of infection in total knee arthroplasty: an algorithm. Knee Surg Sports Traumatol Arthrosc 17:667-675

22. Love C, Marwin SE, Palestro CJ (2009) Nuclear medicine and the infected joint replacement. Semin Nucl Med 39:66-78

23. Tsukayama DT, Goldberg VM, Kyle R (2003) Diagnosis and management of infection after total knee arthroplasty. J Bone Joint Surg Am 85A(Suppl 1):S75-S80

24. Neut D, Van Horn JR, Van Kooten TG, Van Der Mei HC, Busscher HJ (2003) Detection of biomaterial-associated infections in orthopaedic joint implants. Clin Orthop Relat Res 413:261-268

25. Ghanem E, Parvizi J, Burnett RS, Sharkey PF, Keshavarzi N, Aggarwal A, Barrack RL (2008) Cell count and differential of aspirated fluid in the diagnosis of infection at the site of total knee arthroplasty. J Bone Joint Surg Am 90:1637-1643

26. Bergin PF, Doppelt JD, Hamilton WG, Mirick GE, Jones AE, Sritulanondha S, Helm JM, Tuan RS (2010) Detection of periprosthetic infections with use of ribosomal RNA-based polymerase chain reaction. J Bone Joint Surg Am 92:654-663

27. Portillo ME, Salvadó M, Trampuz A, Plasencia V, RodriguezVillasante M, Sorli L, Puig L, Horcajada JP (2013) Sonication versus vortexing of implants for diagnosis of prosthetic joint infection. J Clin Microbiol 51:591-594

28. Choi HR, von Knoch F, Zurakowski D, Nelson SB, Malchau H (2011) Can implant retention be recommended for treatment of infected TKA? Clin Orthop Relat Res 469:961-969
29. Gardner J, Gioe TJ, Tatman P (2011) Can this prosthesis be saved?: implant salvage attempts in infected primary TKA. Clin Orthop Relat Res 469:970-976

30. Kuiper JW, Vos SJ, Saouti R, Vergroesen DA, Graat HC, DebetsOssenkopp YJ, Peters EJ, Nolte PA (2013) Prosthetic jointassociated infections treated with DAIR (debridement, antibiotics, irrigation, and retention): analysis of risk factors and local antibiotic carriers in 91 patients. Acta Orthop 84:380-386

31. Buller LT, Sabry FY, Easton RW, Klika AK, Barsoum WK (2012) The preoperative prediction of success following irrigation and debridement with polyethylene exchange for hip and knee prosthetic joint infections. J Arthroplast 27:857-864

32. Gulhane S, Vanhegan IS, Haddad FS (2012) Single stage revision: regaining momentum. J Bone Joint Surg Br 94(11 Suppl A): $120-122$

33. Whiteside LA, Peppers M, Nayfeh TA, Roy ME (2011) Methicillin-resistant Staphylococcus aureus in TKA treated with revision and direct intra-articular antibiotic infusion. Clin Orthop Relat Res 469:26-33

34. Silva M, Tharani R, Schmalzried TP (2002) Results of direct exchange or debridement of the infected total knee arthroplasty. Clin Orthop Relat Res 404:125-131

35. Nettrour JF, Polikandriotis JA, Bernasek TL, Gustke KA, Lyons ST (2013) Articulating spacers for the treatment of infected total knee arthroplasty: effect of antibiotic combinations and concentrations. Orthopedics 36:19-24

36. Silvestre A, Almeida F, Renovell P, Morante E, López R (2013) Revision of infected total knee arthroplasty: two-stage reimplantation using an antibiotic-impregnated static spacer. Clin Orthop Surg 5:180-187

37. Hsu YC, Cheng HC, Ng TP, Chiu KY (2007) Antibiotic-loaded cement articulating spacer for 2 -stage reimplantation in infected total knee arthroplasty: a simple and economic method. J Arthroplast 22:1060-1066

38. Martínez JL, Baquero F (2002) Interactions among strategies associated with bacterial infection: pathogenicity, epidemicity, and antibiotic resistance. Clin Microbiol Rev 15:647-679

39. Mittal Y, Fehring TK, Hanssen A, Marculescu C, Odum SM, Osmon D (2007) Two-stage reimplantation for periprosthetic knee infection involving resistant organisms. J Bone Joint Surg Am 89A:1227-1231

40. Panni AS, Vasso M, Cerciello S (2013) Modular augmentation in revision total knee arthroplasty. Knee Surg Sports Traumatol Arthrosc 21:2837-2843

41. Vasso M, Beaufils P, Schiavone Panni A (2013) Constraint choice in revision knee arthroplasty. Int Orthop 37:1279-1284

42. Deirmengian C, Greenbaum J, Stern J, Braffamn M, Lotke PA, Booth RE Jr, Lonner JH (2003) Open debridement of acute grampositive infections after total knee arthroplasty. Clin Orthop Relat Res 416:129-134

43. Barberan J, Aguilar L, Carroquino G, Gimenez MJ, Sanchez B, Martinez D, Prieto J (2006) Conservative treatment of staphylococcal prosthetic joint infections in elderly patients. Am J Med 119:993.e7-10

44. Kim YH, Choi Y, Kim JS (2011) Treatment based on the type of infected TKA improves infection control. Clin Orthop Relat Res 469:977-984

45. Azzam KA, Seeley M, Ghanem E, Austin MS, Purtill JJ, Parvizi J (2010) Irrigation and debridement in the management of prosthetic joint infection: traditional indications revisited. J Arthroplast 25:1022-1027

46. Mortazavi SM, Vegari D, Ho A, Zmistowski B, Parvizi J (2011) Two-stage exchange arthroplasty for infected total knee arthroplasty: predictors of failure. Clin Orthop Relat Res 469: 3049-3054 
47. Salgado CD, Dash S, Cantey JR, Marculescu CE (2007) Higher risk of failure of methicillin-resistant Staphylococcus aureus prosthetic joint infections. Clin Orthop Relat Res 461:48-53
48. Volin S, Hinrichs S, Garvin K (2004) Two-stage reimplantation of total joint infections: a comparison of resistant and nonresistant organisms. Clin Orthop Relat Res 427:94-100 